\title{
Study and Simulation for Fuzzy PID Temperature Control System based on ARM

\author{
Guiling Fan ${ }^{1,}$ a and Ying Liü, b \\ ${ }^{1}$ Xijing University, Shaanxi Xi'an, 710123, China \\ a76861639@qq.com, ${ }^{b} 15905721 @ q q . c o m$
}

Keywords: Fuzzy PID; MATLAB; Simulation; Temperature control

\begin{abstract}
This paper studies the fuzzy PID controller design algorithm, and it has set up a hardware platform for the temperature control system thermal analyzer, respectively with MATLAB tools to conventional PID and fuzzy PID control method has carried on the simulation analysis, and in the actual building experiments have been carried out to test system platform. Experimental results show that the fuzzy PID control has good dynamic response and steady state characteristics, with strong anti-interference ability and better robustness and can satisfy the thermal analyzer temperature control system of a wide range of temperature control accuracy requirements.
\end{abstract}

\section{Introduction}

Thermal analyzer temperature control system has the characteristics of nonlinear, time-varying and time lag for the temperature control system of controlled object with these properties, and the conventional PID control method is difficult to meet the requirements of a wide range of temperature control precision. Fuzzy control is a kind of intelligent control method, and it has the characteristics of simple structure. It does not need accurate mathematics model, and it has been widely used in televisions, cameras, washing machines, refrigerators, electric rice cooker, air conditioning, cameras, copiers, and many other aspects.

In order to improve a wide range of temperature control accuracy, this article adopts the method of fuzzy control and PID combination, design implements a fuzzy PID control based on ARM microprocessor thermal analyzer of the temperature control system.

\section{The Fuzzy Control Theory}

The fuzzy control principle is shown in Fig. 1. Fuzzy control system and computer control system of the main difference is usually adopted fuzzy controller.

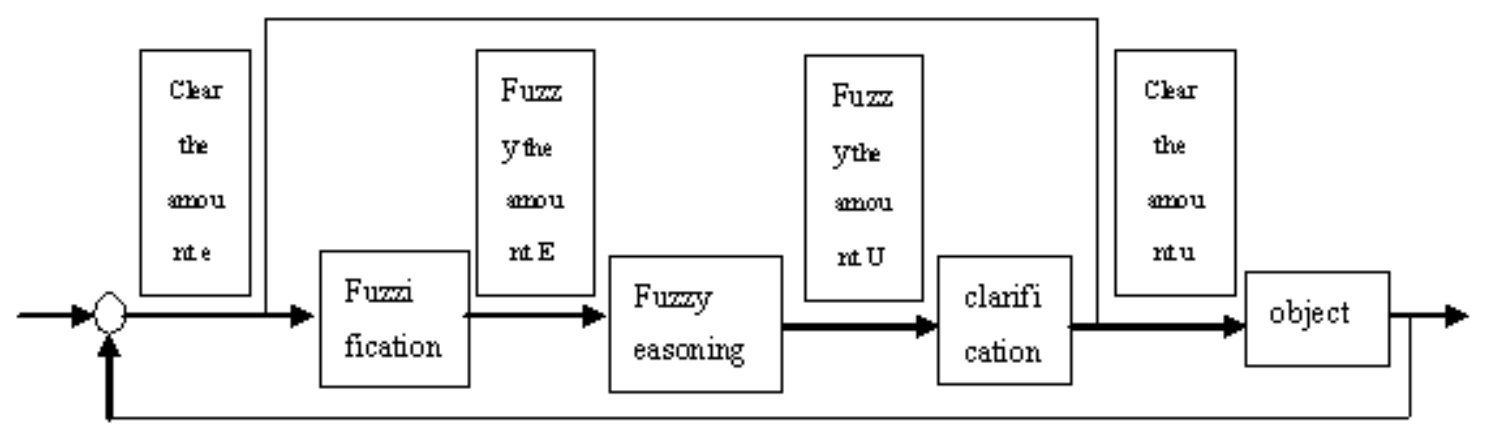

Figure 1. Fuzzy controller

Fuzzy control is made from fuzzy reasoning, reasoning processing and clarifications of three parts. Blur the process state information is the purpose of accurate value of membership function of fuzzy subset into language variables. Fuzzy reasoning is the use of fuzzy knowledge base of fuzzy 
relation, fuzzy reasoning, fuzzy relation equation, and the fuzzy output. Motivation is the subset of fuzzy control that is transformed into the exact control output.

The basic principle of fuzzy control is fuzzy controller by fuzzy of input processing, after a certain language rules of fuzzy reasoning, reasoning. It is concluded that the output of embarrassment, the controlled variable precision output values.

\section{Fuzzy Self-Tuning PID Controller}

Due to the conventional PID controller can't online setting parameters, and the conventional PID controller for nonlinear and time-varying complex system and the model is not clear that the system cannot well control. The PID parameters is not setting a very difficult or not setting, which sometimes can't get the expected control effect. So it does not have integral link and simple fuzzy controller, and it is difficult to completely eliminate the steady-state error in a fuzzy control system. There often will be a small oscillation phenomenon near the balance in the case of variable classification that is not enough. But the fuzzy controller and the model of complex system that can not clear carry out simple and effective control, so combining both fuzzy control and PID control. In this way, it both has the advantages of fuzzy control which is flexible and adaptable, and it has the characteristics of high precision of PID control.

The fuzzy technology and PID control algorithm combining the fuzzy PID controller has a variety of methods. One fuzzy controller is used to give online self-tuning PID controller (or since the correction, self-tuning) PID parameters, and it constitutes fuzzy self-tuning or self-tuning PID controller parameters, another kind is in the big deviation within the scope of using proportional control, and fuzzy control in small deviation. Two kinds of control mode switch is to control according to predetermined deviation threshold, and this will constitute a fuzzy -p dual-mode piece wise controller. Similarly, the multimo de subsection control algorithm, can design according to different conditions and requirements section with different modal control. This system uses the first method, the use of fuzzy control for online self-tuning PID.

According to the output of fuzzy inference machine direct physical meaning, a fuzzy PID controller can be divided into: gain gather, direct control of volume type and mixed type. Although there are many kinds of structure forms a fuzzy PID controller, and basically working principle is same. That is the basic principle and method of fuzzy mathematics, the rules of the condition and operation in fuzzy sets, the fuzzy control rules and related information as the repository of knowledge into computer, then the computer according to the actual response of the fuzzy reasoning system, which can realize the best adjustment of PID parameters. The structure of the more common fuzzy PID controller is shown in Fig. 2.

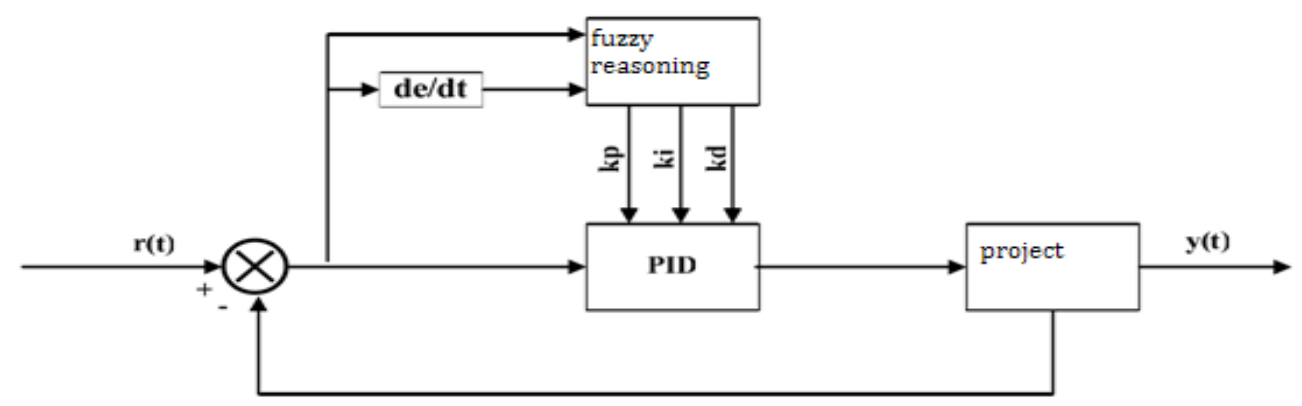

Figure 2. The basic structure of fuzzy self-tuning PID controller

The basic idea of fuzzy PID controller is that first find out the three parameters of PID deviation e between the change of the ec deviation, the fuzzy relationship and detection e continuously in the run. Then according to the principle of fuzzy control we amend the three parameters row change, and in order to meet the different e and ec. According to the principle of fuzzy control to the three 
parameters row change. It meets the different e and ec, to the different requirement of the control parameters. According to the parameters $\mathrm{kp}, \mathrm{Ki}$ and $\mathrm{Kd}$ use, under the different $\mathrm{e}$ and ec, on the parameters of PID controller.

\section{The MATLAB Simulation}

\section{Controlled Object as a Pure Lag Model:}

$$
G(s)=\frac{1.5 e^{-30 s}}{145 s+1}
$$

In the MATLAB command window to run a Fuzzy into Fuzzy logic editor, and establish a new file. We select the types of control model, and it is based on the above analysis input respectively $\mathrm{E}, \mathrm{EC}, \Delta \mathrm{KP}, \Delta \mathrm{Ki}, \Delta \mathrm{Kd} . \mathrm{K}$ of membership According to the above article 49 fuzzy control rules to complete the control input, functions and quantitative range.

Establishes a system files. After completing fuzzy controller file editor, it is saved to a folder. It is established the fuzzy self-tuning PID control system model [36, 37, 38] in SIMULINK, as shown in Fig. 3.

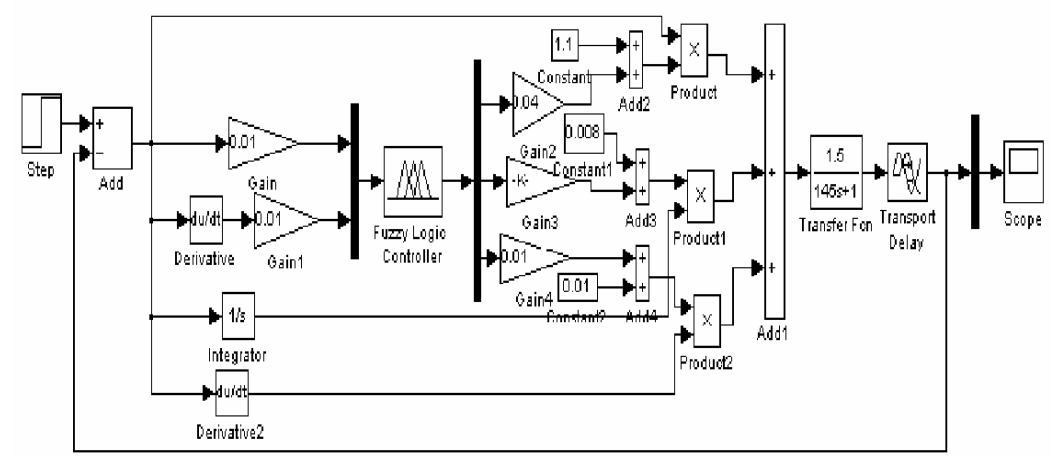

Figure 3. Fuzzy self-tuning PID control system model

The thermal analysis instrument is mainly used for heating furnace, high temperature control, and general control temperature is not lower than $500{ }^{\circ} \mathrm{C}$.To observe in the control effects under different control mode, the temperature control experiment uses conventional PID control and fuzzy PID control in two ways.

The design adopts thermal analysis instrument heating furnace temperature control experiments, as shown in Fig. 4.

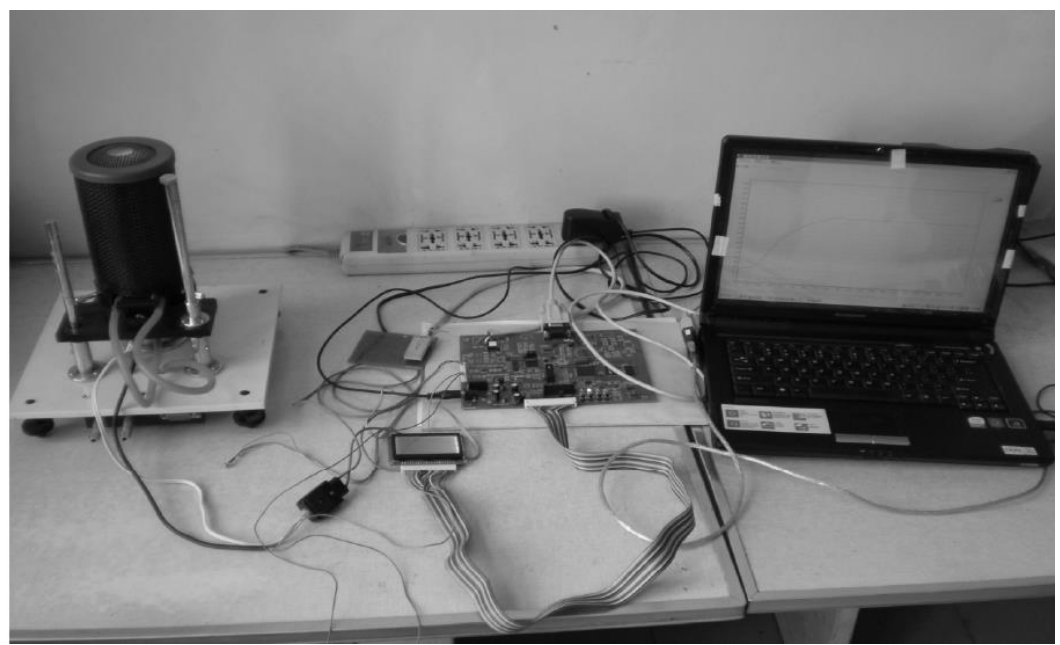

Figure 4. Practicality picture of thermal analyzer temperature control system 


\section{Conventional PID and Fuzzy PID Simulation Results Are Shown in Fig. 5.}

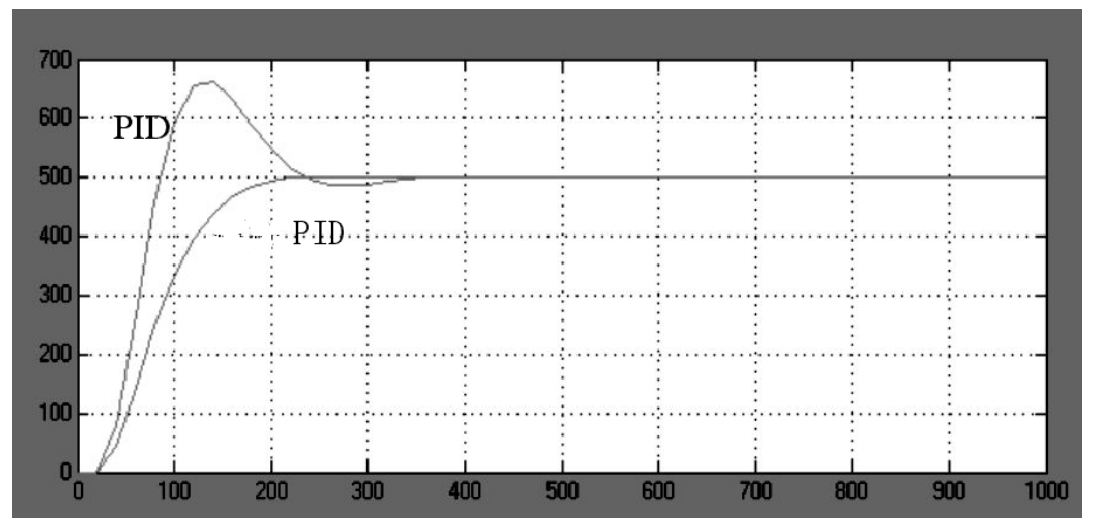

Figure 5. Simulation results

It can be seen from the simulation results that the fuzzy self-tuning PID control has a smaller overshoot and the regulation of small amount of time. It has a good dynamic response and steady state characteristics, and the advantages of high accuracy. And it has good anti-jamming performance and good robustness. Obviously, the fuzzy self-tuning PID control is a kind of simple design, convenient for implementation and optimization of control rules and the intelligent controller with excellent performance has good dynamic performance, steady state characteristics of high precision.

Using MATLAB simulation research on the fuzzy PID control method, and then this temperature control system, a experiment is carried out by the test results that can be concluded that the control system can coordinate work of software and hardware, the application of the fuzzy PID control method can meet requirements for temperature control in thermal analyzer.

\section{Summary}

In this paper, we study the rule of fuzzy PID control, and temperature control system for thermal analyzer has the characteristics of nonlinear, time-varying and time lag, the conventional PID control method is difficult to meet the requirements of a wide range of temperature control accuracy. Therefore the combination of fuzzy control and PID method, design and implements a fuzzy PID control of the thermal analyzer based on ARM temperature control system. Experimental test results show that the system is reliable, stable work, and it can better meet the requirements of high control precision of the system.

\section{References}

[1] T.C.Callai, J.E.S.Santos, R.R.Sumar, etc. Applying the Potentiality of Using Fuzzy Logic in PID Control Design [J]. Advances in Soft Computing,2013,1:193-204

[2] Taifu Li, Yingying Su, Bingxiang Zhong. Remodeling for Fuzzy PID Controller Based on Neural Networks [J]. Fuzzy Information and Engineering(ICFIE),ASC 40,2011,714-725

[3] Kevin M.Passino, Stephen Yurkovich. Fuzzy Control [M]. BeiJing: Tsinghua University Press, 2011

[4] Jong-kwon Kim1, Soo-hong Park, TaeSeok Jin. Simplified Fuzzy-PID Controller of Data Link

[5] Antenna System for Moving Vehicles [J]. PRICAI 2010,LNAI 4099:1083-1088

[6] Taifu Li, Jundi Xiong, Rui Zhang, etc. Hardware implementation of fuzzy PID controllers [J]. 
[7] Fuzzy Optimization and Decision Making ,2010,5:113-122

[8] B.M.Mohan, Arpita Sinha. The simplest fuzzy PID controllers: mathematical models and stability analysis [J]. Soft Computing ,2010,10:961-975

[9] NXP Semiconduetors. LPC2364/66/68/78 User manual. 2011

[10]FairehildSemieonductorCorporation.74HC574DataSheet.2010

[11]Integrated Deviee Teehnology, Ine.74LVCH162245DataSheet.2010

[12]Analog Deviees.Ine.ADG506ADataSheet.2010

[13]Burr-BrownCorporation.DAC712DataSheet.2010 